\title{
STRONG GRAVITATIONAL LENSING ON THE HUBBLE DEEP FIELD
}

\author{
R. D. BLANDFORD \\ 130-33 Caltech, \\ Pasadena, \\ CA 91125, USA
}

\section{Introduction}

The Hubble Deep Field, henceforth HDF, (Williams et al 1996) is a unique data set for studies of faint galaxies. A small area was chosen well away from known local density enhancements and sources of obscuration. It was imaged for 30 complete orbits in each of four filters centered on wavelengths $\lambda 300,450,606,814 \mathrm{~nm}$. There are three, contiguous WFPC2 frames and a solid angle $15,500 \operatorname{arcsec}^{2}$ is usable. This tiny region is teeming with galaxies. At least 2500 significant features can be identified down to $R=30$, though it is not clear that these are all entire galaxies.

This data archive has, as intended, spawned a variety of follow up studies, including infrared and radio surveys and a redshift survey using the Keck telescope. This last program has garnered over 500 redshifts on the HDF and neighboring "flanking fields" (Cohen et al. 1996, Phillips et al. 1997), of which over 100 are on the HDF itself and therefore imaged with unprecedented depth. The redshift sample is essentially complete to $R \sim 23$.

\section{Some Key Results}

For completeness, let me list some developments that have come from analysis of the HDF. Some of these are original discoveries; others are verifications and elaborations of prior results. All of them are discussed in a forthcoming proceedings of a conference held at STScI ("The Hubble Deep Field", ed. Livio \& Ealley).

- There appear to be $\sim 100$ billion faint sources observable over the whole sky. These may be galaxies, but are surprisingly blue in color and they appear to be very compact with characteristic sizes $\sim 3 h^{-1} \mathrm{kpc}$. 
- The slope of the galaxy counts flattens fainter than $B \sim 25$ and most of the galaxian contribution to the brightness of the night sky comes from around this magnitude. (Again some caution is necessary because HST may not be sensitive to extended low surface brightness regions.)

- Perhaps the most significant discovery is of a substantial population of normal galaxies at redshifts $z \sim 3$. These are about 3 percent of the $\sim 25^{m}$ galaxies and represent a major breakthrough in the study of galaxy formation (Steidel et al. 1996, Lowenthal et al. 1997).

- It has also been found that just under half of galaxies with $z>0.5$ are irregular morphologically, a far higher proportion than is the case at lower redshift (Abraham, these proceedings).

- The redshift surveys have shown that galaxies with $z \sim 0.5$ are less clustered than locally. In addition, about 40 percent of these are concentrated within narrow features in redshift space (Cohen et al. 1996). (As we only have a pencil beam survey, with linear width much smaller than the two point correlation function, this finding must be interpreted judiciously.) The redshift survey has also allowed a convincing demonstration of the reliability of the color-redshift method, at least for $z<1, R<24$ (Hogg et al. 1997 preprint).

\section{The Number Problem}

That there are too many faint galaxies may be demonstrated succinctly, if somewhat polemically. From the measured local luminosity function there are roughly $\sim 4 \Phi^{*}$ galaxies with luminosity in excess of $\sim 0.01 L^{*}$. This evaluates to $\sim 0.4$ billion galaxies (BG) per Hubble volume. Now, in an Einstein-De Sitter universe, there are roughly four Hubble volumes out to $z \sim 3$, which is as far as we are likely to see faint galaxies in the optical and therefore we only expect to see $\sim 1.6$ BG that are counterparts of the bright galaxies that we see around us. There are therefore roughly 60 times more faint objects than there ought to be.

There are a variety of resolution of this problem. In ascending importance these include:

- Denial. A surprisingly large number of cosmologists still see no problem.

- Incompetence. We may have underestimated the local luminosity function and may be missing many low surface brightness galaxies (McGaugh 1994). There is some evidence that this may be the case at the level of a factor 2, but this can hardly be the total explanation.

- Cosmography. Open universes give us more comoving volume before Lyman limit absorption extinguishes the galaxies. However, they do this at the expense of increasing the luminosity distance and mak- 
ing the same galaxies much fainter. We have to appeal to a highly non-standard world model to find enough help here. This would be a wonderful discovery, if true.

- Local dwarfs. The problem here is that the local faint luminosity function appears to be quite flat, although it does appear to steepen at fairly modest redshifts. This is unlikely to be the whole explanation because cluster lensing (and related arguments) tell us that a sizable fraction of the faint galaxies have to be at high redshift.

- Fading population. There may be a large population of intermediate luminosity galaxies with $z \sim 1$ which form and have too shallow potential wells to retain their gas in the face of the supernova blast waves associated with the first generation of stars. These galaxies should still be visible locally in the infrared (Babul \& Feguson 1996). Unfortunately, we see no evidence for them (Hogg et al. 1997).

- Mergers - the topic of this meeting. The faintest, $30^{m}$ objects may be the building blocks out of which galaxies are being formed. If so, then it is surprising that we do not see them more clustered (Colley et al. 1997). However, this may not be a fatal objection because the merging time can be quite short.

\section{Strong Gravitational Lensing}

These prefatory remarks provide a context for an ongoing investigation of strong gravitational lensing on the HDF. This is also motivated by radio and other surveys which are showing that a fraction $\sim 0.002$ of high redshift sources are multiply-imaged by intervening galaxies. On this basis, Hogg et al. (1996) argued that there should be $\sim 5-10$ strong lenses on the HDF and identified the most promising candidates on morphological grounds. Unfortunately, it has proved hard to follow this up as the candidates are very faint for spectroscopic study. There are still no convincing examples of multiply-imaged galaxies on the HDF. I have therefore tried another approach to attempt to locate these gravitational lenses or argue that they are not present, (which would immediately imply that the faint galaxy sources are predominantly relatively close by).

What I have tried to do is to combine the exquisite imaging of the potential lens galaxies on the HDF with their measured redshifts to identify the galaxies most likely to be lenses and then to use the faint galaxy counts to quantify the lensing probability. In addition I have performed simulations to see if it is possible to detect multiple imaging of a background against the light from the lens galaxy. As this research is ongoing, I can only report preliminary conclusions. 


\subsection{LENSES}

As these are field galaxies with no discernible cluster along the line of sight, multiple imaging will be confined to the central regions of the most concentrated galaxies and least affected by dark matter in the halo. Now for every galaxy in the redshift sample, we can use the redshift to estimate the surface brightness in the frame of the source in the emitted B band. To accomplish this, we use the $F 814 W$ filter, (as this image will be least susceptible to the effects of dust), and make a spectral correction based upon the integrated color. (This is preferable to performing the spectral correction on a pixel by pixel basis because we are interested in the global mass distribution.) We next convert this surface brightness into a surface mass density by assuming a constant mass to light ratio in the B-band. Local measurements of $h M / L_{\mathrm{B}}$ suggest a value of 5 for spirals and 10 for ellipticals. Measurements of known gravitational lenses (which may be a biased sample) suggest twice this value (Keeton, Kochanek \& Falco, 1997 preprint). (I included irregulars and unclassified galaxies in with the spirals.) Both observations and the expectations from passive stellar evolution indicate that galaxies were about twice as luminous at $z \sim 1$ and a simple, empirical evolutionary correction was made. We must next make some cosmographic assumptions. For illustration, I shall adopt an open universe with $\Omega_{0}=0.3$. (The outcome is independent of $h$.) I shall also place all the faint sources at $z=3$. The next step is to adopt the surface brightness maps of each of the 114 galaxies with known redshifts $z<1.5$ and solve Poisson's equation using a Fourier method to obtain the equivalent surface potential. This can, in turn, be converted into a deflection vector field and a magnification field, obtained from the gradient of the deflection field. Images with negative magnification are multiple and so we can compute the cross section for multiple imaging by projection back to the source plane. There were many surprises. The first was that most galaxies do not multiple image, at least at the resolution of $H S T$. (Of course if every galaxy contains a massive black hole in its nucleus, they will each multiple image every other galaxy an infinite number of times (Rauch \& Blandford 1994). However, this is is not a concern for observational astronomy!) Those that do are almost all elliptical galaxies that have higher central surface brightness and are assumed to have higher mass to light ratio. In fact, three-quarters of the cross section is contributed by four galaxies. Secondly, the propensity for multiple imaging is strongly dependent upon the mass to light ratio. For example, increasing the central mass to light ratio for local galaxies from 10 solar units to 20 solar units increases the cross section of the strongest lens from $\sim 0.3 \operatorname{arcsec}^{2}$ to $\sim 0.9 \operatorname{arcsec}^{2}$. As always, finding gravitationally lensed sources of known redshift is a very sensitive measurement of the 
central mass to light ratio of the lensing galaxy. Thirdly, the four strongest lenses all have $z \sim 1$ and $L \sim L^{*}$ and are therefore quite ineffectual if the sources have redshifts much less than $z \sim 2$. Finding multiple images systematically from a complete sample of lens galaxies is a potentially powerful probe of the faint source redshift distribution. The integrated cross section over the whole HDF is surprisingly low, only $\sim 1 \operatorname{arcsec}^{2}$ for the "local" choice of $M / L$ increasing to $\sim 3 \operatorname{arcsec}^{2}$ for the lens-normalized choice.

\subsection{SOURCES}

The sources were classified by size rather than integrated flux. Size was defined operationally as the number of contiguous 0.04 arcsec pixels with counts above $2.5 \sigma$. There are $\sim 1600$ galaxies with sizes between 5 and 50 pixels (about two-thirds of the total number of galaxies). The median galaxy in this sample has a size of 13 pixels. As we extrapolate to smaller images, we do not expect to find a large increase in the potential source density unless there is a sharp upturn in the magnitude source counts below $\sim 30^{m}$. The total density of source galaxies is therefore $\sim 0.1 \operatorname{arcsec}^{-2}$ and the total probability of finding one of them multiple-imaged if they are at redshift $z \sim 3$ is $\sim 0.1$ assuming the local $M / L$ and $\sim 0.3$ for the lensnormalized $M / L$. For the individually strongest galaxies, the probabilities per lens are therefore $\sim 0.03$ and $\sim 0.1$ respectively.

\subsection{DETECTABILITY}

What is clear from the foregoing discussion is that it may pay to seek multiple images very carefully in judiciously chosen locations, rather than by inspecting a large sample of galaxies. Now, it goes without saying that this is a daunting task. What we are looking for are faint, inclined arc-like features within $\sim 5 \mathrm{kpc}$. They are likely to be patchy and blue. In other words, they look just like spiral arms! Now, I believe that the prospects are not so bleak if one has images with a resolution and depth comparable with those on the HDF in several filters. In particular, ellipticals are clearly preferred and can be identified on spectral and not just morphological grounds. They have relatively smooth light profiles against which arcs can be brought out by image processing techniques. Furthermore the image pattern that is expected, and can be easily simulated, is quite distinctive. The magnified images should have similar colors although variable reddening by the lens galaxy can confound this expectation.

A quick inspection of the images of all of the galaxies in the redshift survey reveals several features that could be formed by galaxy lensing. However, none of these is convincing, based upon the criteria listed above. Looking at the four best candidates, only the strongest of these exhibits a 
feature that is worth considering further and this is a large, tangential arc at a radius of $\sim 1$ arcsec. It has a similar color to the rest of the galaxy and would require $h M / L \sim 40$ solar units. It is far more likely to be a shell produced by tidal stripping of a merging galaxy.

\section{Conclusion}

Using the observed surface brightnesses of galaxies on the HDF, we estimated a strong lensing frequency at least an order of magnitude lower than that based on radio surveys and advertized in Hogg et al. 1996. If this is the correct approach to estimating the frequency of multiple imaging, then it should not be a surprise that we have no good examples to offer as yet. Put another way, the best multiple image candidates selected from the HDF on morphological grounds, required $M / L$ ratios far in excess of those measured in local, normal galaxies. Perhaps, in retrospect, this is quite reasonable. Many of the secure examples of gravitational lensing are unusual because they belong to a rich compact group or cluster which magnifies the focusing power of an individual galaxy and the HDF was selected, as far as possible, to avoid such concentrations. Furthermore, the source population in the radio surveys is known to be at high redshift, whereas not all of the faint optical sources may be distributed in this manner.

Nevertheless, in spite of this somewhat negative, interim report, I hope that I have demonstrated that scrutiny of deep images in a larger sample of bright, high redshift, field ellipticals should tells us much about both the evolution of their mass to light ratios and the nature and distances of the very faintest optical sources on the sky.

\section{Acknowledgements}

I thank my colleagues Tereasa Brainerd, David Hogg and Tomislav Kundić for collaboration on various parts of this investigation and Roland van der Marel for consultation on mass to light ratios. Special thanks are due to Judith Cohen who has led the Caltech-Keck redshift survey and colleagues at UCSC and UH who have also contributed many of the redshifts. Acknowledgement must also be made of Bob Williams who had the foresight to see the long term value of the HDF project. Support under NSF grant AST 95-29170 is gratefully noted.

\section{References}

Babul, A. \& Ferguson, H. C. ApJ 458100

Cohen, J. G. et al. 1996 ApJ 471 L5

Colley, W. N. et al. 1997 ApJ 488579

Hogg, D. W. et al. 1996 ApJ 467 L73 
Hogg, D. W. et al. 1997 AJ 113474

Lowenthal, J. D. et al. 1997 ApJ

McGaugh, S. S. 1994 Nature 367538

Phillips, A. C. et al. 1997 ApJ in press

Rauch, K. P. \& Blandford, R. D. ApJ 42146

Steidel, C. C. et al. 1996 AJ 112352

Williams, R. E. et al. 1996 AJ 1121335 